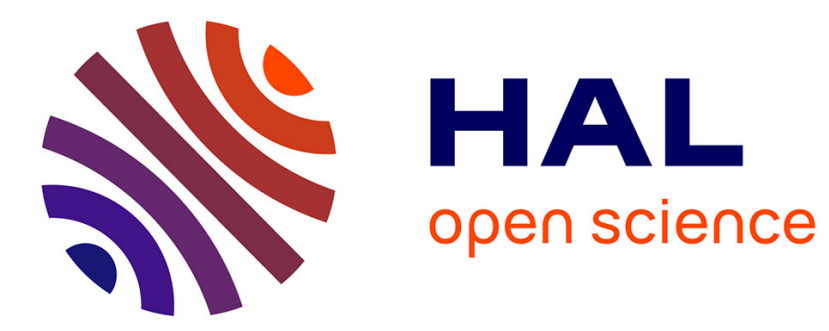

\title{
The relationship between motor deficit and primary motor cortex hemispheric activation balance after stroke: longitudinal fMRI study
}

Cinzia Calautti, P Simon Jones, Marcello Naccarato, Nick Sharma, Diana Day, Ed Bullmore, Elizabeth Warburton, Jean-Claude Baron

\section{To cite this version:}

Cinzia Calautti, P Simon Jones, Marcello Naccarato, Nick Sharma, Diana Day, et al.. The relationship between motor deficit and primary motor cortex hemispheric activation balance after stroke: longitudinal fMRI study. Journal of Neurology, Neurosurgery and Psychiatry, 2010, 81 (7), pp.788. 10.1136/jnnp.2009.190512 . hal-00557415

\section{HAL Id: hal-00557415 \\ https://hal.science/hal-00557415}

Submitted on 19 Jan 2011

HAL is a multi-disciplinary open access archive for the deposit and dissemination of scientific research documents, whether they are published or not. The documents may come from teaching and research institutions in France or abroad, or from public or private research centers.
L'archive ouverte pluridisciplinaire HAL, est destinée au dépôt et à la diffusion de documents scientifiques de niveau recherche, publiés ou non, émanant des établissements d'enseignement et de recherche français ou étrangers, des laboratoires publics ou privés. 


\title{
The relationship between motor deficit and primary motor cortex hemispheric activation balance after stroke: longitudinal fMRI study
}

\author{
C. Calautti ${ }^{1}$, PS Jones ${ }^{1}$, M. Naccarato ${ }^{1}$, N. Sharma ${ }^{1}$, DJ Day ${ }^{1}$, ET Bullmore ${ }^{2}$, \\ EA Warburton ${ }^{3}, \mathrm{~J}-\mathrm{C}$ Baron $^{1 *}$
}

1. Stroke Research Group, Dept of Clinical Neurosciences

2. Wolfson Brain Imaging Centre and Brain Mapping Unit, University of Cambridge, Cambridge, UK.

3. Stroke Unit, Dept of Medicine, Addenbrooke's Hospital, Cambridge

* Corresponding author

Dept Clinical Neurosciences

Addenbrooke's Hospital Box 83

Cambridge CB2 2QQ, UK

jcb54@cam.ac.uk

Acknowledgements: Work supported by MRC Grant G0001219. PS Jones was supported by the Biomedical Research Centre Grant, and N. Sharma by the Stroke Association (Grant TSA 2003/10). .

\section{Licence for Publication:}

The Corresponding Author has the right to grant on behalf of all authors and does grant on behalf of all authors, an exclusive licence (or non exclusive for government employees) on a worldwide basis to the BMJ Publishing Group Ltd to permit this article (if accepted) to be published in PMJ and any other BMJPGL products and sublicences such use and exploit all subsidiary rights, as set out in our licence. 


\section{Abstract}

Background: In the chronic stage of stroke, previous work has shown that the worse the hand motor deficit the greater the shift of primary motor cortex $\left(\mathrm{M}_{1}\right)$ activation towards the contralesional hemisphere (i.e., unphysiological). Whether the same relationship applies at an earlier stage of recovery in serially studied patients is not known.

Methods: fMRI of fixed-rate auditory-cued affected index finger tapping was obtained at 2 time points (mean 36 and 147 days post stroke) in a cohort of 9 ischaemic stroke patients (age: $56 \pm 9$ yrs; 3 women/6 men; 7 subcortical, 1 medullary and 1 cortical). On each fMRI day the unaffected/affected ratio of maximal index tapping rate (IT-R) was obtained. To assess the $\mathrm{M}_{1}$ hemispheric activation balance, we computed the classic Laterality Index (LI). The correlation between LI and IT-R was computed for each time point separately.

Results: The expected correlation between LI-M $\mathrm{M}_{1}$ and IT-R, i.e. motor performance worse with more unphysiological LI, prevailed at both time points (Kendall $p=0.008$ and 0.058 , respectively), with no statistically significant difference between the two regressions. The same analysis for the dorsal premotor cortex and the supplementary motor area showed no significant correlation at either time-point.

Conclusion: These results from a small cohort of longitudinally-assessed patients suggest that the relationship between $\mathrm{M}_{1}$ laterality index and hand motor performance appears independent of time since onset of stroke. This in turn may suggest that attempting to restore the hemispheric balance by enhancing ipsilesional $\mathrm{M}_{1}$ and/or constraining contralesional $\mathrm{M}_{1}$ activity may have consistent efficacy throughout recovery. 


\section{Introduction}

Functional imaging has documented dynamic reorganisation of large-scale cortical motor networks after stroke underlying recovery of function ${ }^{1}$. The Laterality Index (LI $)^{2}$, which reflects the hemispheric balance of activation for a pair of transcallosally-connected areas, is a particularly sensitive marker of concomitant motor deficit. Thus, fMRI studies in chronic stroke patients with variably recovered motor deficit have reported a significant correlation between the degree of hand motor impairment and the LI for the primary motor cortex $\left(\mathrm{M}_{1}\right)$, such that the worse the deficit the more unphysiological the $\mathrm{LI}^{1-4}$. Among these studies, ours had the largest sample $(n=19)^{3}$; it used index finger maximum tapping speed as clinical marker of cortico-spinal tract damage; and the correlation observed did not depend on infarct location, i.e. cortical or not. Consistent with these observations, two studies have reported that the improvements in hand function following specific training were associated with statistically significant returns of the $\mathrm{LI}_{-} \mathrm{M}_{1}$ towards more physiological values ${ }^{5,6}$. In contrast to these clearcut findings concerning M1, our previous $s \operatorname{sudy}^{3}$ found no correlation between motor deficit and the LI for the Dorsal Premotor cortex (PMd) and the Supplementary Motor area (SMA), the main secondary cortical motor areas. This difference between M1 on one hand, and PMd and SMA on the other, was interpreted as being due to anatomical differences between these regions, namely paucity of the transcallosal connections for M1 relative to PMd and SMA, making function of the two primary motor cortices relatively independent from each other, while the PMd and the SMA are more bilaterally organized ${ }^{3}$.

Due to their notorious difficulties, only few longitudinal natural history (i.e., non-interventional) studies have assessed the changes in brain activation patterns during executed movement as recovery proceeds ${ }^{7-15}$. Among these, all three studies that assessed the $\mathrm{LI}^{10-12}$ reported a significant increase towards normality of the LI-M paralleling recovery, and two reported a trend for a correlation between changes in LI$\mathrm{M}_{1}$ and concomitant clinical improvements ${ }^{10,11}$. However, no study so far has assessed whether the above-described correlation between the LI-M $\mathrm{M}_{1}$ and hand motor function is consistent over time. A consistent relationship would suggest that attempting to restore the hemispheric balance by enhancing ipsilesional M1 and/or 
constraining contralesional M1 activity ${ }^{1}$ could be applied across recovery. The converse finding would suggest this therapeutic approach should be envisaged only for the time window when the relationship is present.

Here we investigated whether the correlation between the LI-M ${ }_{1}$ and hand motor performance we previously reported for the chronic stage ${ }^{3}$ is also present at an earlier stage of recovery in the same patients studied longitudinally. Based on the above, our hypothesis was that this relationship would not be different between the two time-points. The PMd and SMA were also assessed, but here the hypothesis was that there would be no correlation between the LIs and finger tapping performance at either

time-point. 


\section{Patients and Methods}

\section{Patients}

Out of our previously reported sample of 19 patients studied with fMRI in the chronic stage $^{3}$, there were nine patients who had had an fMRI study at an earlier stage of recovery according to a longitudinal design. These nine patients (age: $56 \pm 9$ yrs; 3 women/6 men) form the clinical material for the present report.

Patients were selected based on i) first-ever stroke; ii) $\mathrm{M}_{1}$ hand area of the primary motor cortex intact or largely preserved based on late structural MRI; iii) ability to perform the fMRI motor task (see below); iv) right-handedness; and v) age over 40yrs. Exclusion criteria were i) any cognitive impairment impairing full cooperation; ii) silent infarct or significant small vessel disease on admission CT; iii) ipsilateral synkinesia or proprioceptive loss; and iv) current intake of drugs that may interfere with recovery such as psychotropics. A post-hoc exclusion criterion was motor deficit lasting less than two weeks, as in this study we were interested in patients with more prolonged impairment. All patients received standard physiotherapy. The Regional Ethics Committee approved the study and written informed consent was obtained.

\section{Methods}

To allow for meaningful comparison with our previous report ${ }^{3}$, we implemented exactly the same previously detailed methodology ${ }^{3}$, which will be only summarised here.

\section{Motor perfomance}

On the day of scanning, the maximum number of index finger-to-thumb taps in 15 seconds (IT-Max) was obtained for both the affected and unaffected hands. In order to control for global physiological changes in tapping rate from subject to subject and from day to day in the same subject, we normalized the motor performance on the 
affected-side by that of the unaffected-side ${ }^{4,16}$, by computing an "index-tapping ratio" (IT-R) as IT-Max unaffected $_{\text {ITT-Max }}$ affected $^{3}$.

\section{fMRI scanning paradigm.}

Block-design fMRI was performed under two conditions, each replicated 4 times and performed in pseudo-random and balanced order: 1) affected-hand index-thumb tapping, auditory cued at a frequency of $1.25 \mathrm{~Hz}$; and 2) rest, with the auditory tones delivered at the same frequency. For both conditions, the subject kept the eyes closed and the head was gently immobilized in a dedicated headrest. Instructions ("Move" or "Rest") were given aurally. Just prior to scanning, subjects were instructed and trained into the task using the same stimulation software until they were able to adequately follow the cues and instructions. Exactly the same task and paradigm were applied at both time-points.

\section{Data acquisition.}

We used a $3 \mathrm{~T}$ Bruker Medspec scanner (Ettlingen, Germany). T2*-weighted, echoplanar images (EPI) were acquired with $\mathrm{TR}=3 \mathrm{~s}$. Activation blocks lasted 24 seconds (8 EPI volumes) and the Rest blocks lasted 12 seconds (4 EPI volumes), with 21 noncontiguous axial planes (thickness $=5 \mathrm{~mm}$; matrix size $64 \mathrm{x} 64$ ) parallel to the AC-PC line. In total 54 images were acquired for each session, but the first six images were discarded to allow for $\mathrm{T} 1$ equilibration effects, leaving a total of 48 images per session. T2-weighted structural images ( $3 \times 3 \times 4 \mathrm{~mm}$ voxels) were also obtained for anatomical orientation.

\section{Image Processing.}

We used Statistical Parametric Mapping (SPM2; Wellcome Department of Imaging Neuroscience, London, UK). Pre-processing steps comprised slice acquisition time correction, within-subject image realignment, spatial normalization to the MNI template (voxel size: 4 x 4 x $4 \mathrm{~mm}$ ) and spatial smoothing using a 12-mm Gaussian kernel. Masking was not necessary as the lesions were not large and did not cause cavitation and/or brain distortion in any patient. Data sets were rejected if head displacement was >2mm; no patient in this longitudinal investigation had to be excluded because of this. The time series was high-pass filtered to remove low- 
frequency noise, and the default temporal frequency cut-off was used. Single-subject fixed-effect t-maps (Task $>$ Rest) were generated using default $\mathrm{p}<0.001$ uncorrected.

\section{Regions of Interest.}

The LI was obtained for M1, PMd and SMA, within Regions of Interest (ROIs) applied onto each hemisphere. These ROIs have been described in detail earlier ${ }^{3}$. Briefly, the $\mathrm{M}_{1}$ and PMd ROIs were extracted from the wfu_Pickatlas template ${ }^{17}$ with slight modifications (see below), while the SMA ROI was extracted unchanged from the AAL template ${ }^{18}$. The boundaries for the M1 ROI were the central sulcus posteriorly, $\mathrm{z}=24 \mathrm{~mm}$ inferiorly, and the middle of the precentral gyrus anteriorly (except in its cranial part where it occupies most of the precentral gyrus). The boundaries for the PMd ROI were M1 posteriorly, $\mathrm{z}=51 \mathrm{~mm}$ inferiorly, and the anterior limit of the Pickatlas premotor ROI anteriorly. The ROIs were automatically defined on each subject's spatially normalised data set,

\section{Laterality Index}

The method has been described in detail elsewhere ${ }^{3}$. Briefly, the ROIs (i.e., the $\mathrm{M}_{1}$, PMd and SMA ROIs for each side of the brain) were applied onto the single-subject fixed-effects $\mathrm{t}$-maps. For each ROI, a sum of $\mathrm{t}$ values $\left(\sum \mathrm{t}\right)$ was computed across voxels with positive activation as described previously ${ }^{3}$. The weighted LI (wLI) ${ }^{3}$ was calculated for each ROI according to:

$$
\mathrm{wLI}=\left(\Sigma \mathrm{t}_{\mathrm{I}^{-}}-\Sigma \mathrm{t}_{\mathrm{C}}\right) /\left(\Sigma \mathrm{t}_{\mathrm{I}}+\Sigma \mathrm{t}_{\mathrm{C}}\right)
$$

, where $\mathrm{C}$ and I reflect the ipsilesional and contralesional ROIs, respectively. Based on this equation, a wLI of +1 reprersents completely lateralized activation towards the ipsilesional hemisphere, and a wLI of -1 reflects completely unphysiological activation fully lateralized to the contralesional hemisphere.

\section{Statistical analysis}

The correlation between the wLI for each ROI and the IT-R was assessed at each time point using Kendall's non-parametric test. Based on previous reports and our hypothesis (see Introduction), one-tailed p<0.05 was considered significant for the LI$\mathrm{M}_{1}$ vs IT-R correlation. As no significant correlation was expected for PMd and SMA 
vs IT- $\mathrm{R}^{3}$, two-tailed $\mathrm{p}<0.05$ was considered significant. Comparison of the correlations obtained at Session 1 and Session 2 used Pearson's test.

\section{Results}

Table 1 lists the main demographics and clinical and motor scores at both sessions for the 9 patients. The strokes were subcortical in 7, medullary in 1 and cortical in 1 patient. Time from stroke onset was $36 \pm 14$ (range 10-60) and $147 \pm 44$ (range 90-230) days at Sessions 1 and 2, respectively. There was a significant improvement in affected-hand IT-Max between the two sessions ( $p=0.007$ Wilcoxon), but not in unaffected-hand IT-Max. There was also a significant decrease in IT-R $(\mathrm{p}=0.03)$.

There was a significant increase in wLI-M $_{1}$ between the two sessions ( $\mathrm{p}=0.05)$, but not for wLI-SMA or wLI-PMd (Table 1). To illustrate visually these changes in activation patterns over time, Figure 1 depicts activation maps averaged across the 9 subjects of the cohort at time-point 1 and time-point 2, showing more bilateral M1 activation at the former than the latter time-point.

The relationship between LI-M 1 and IT-R at both sessions is shown in Figure 2A. There was a significant negative correlation at Session 1 (Kendall's tau $=-0.648$, $1 \mathrm{p}=0.008)$ and a nearly-significant trend at Session $2(\operatorname{tau}=-0.423,1 \mathrm{p}=0.058)$. There was no significant difference between these two correlations. To illustrate further these findings, Figure 2B presents the previously published IT-R vs wLI-M I $_{1}$ scatterplot for the sample of 19 chronic-stage patients ${ }^{3}$, augmented by the nine data points from Session 1 reported here. This illustrates the highly significant relationship between index tapping speed and the laterality index for $\mathrm{M}_{1}$ across 28 measurements, including those from Session 1 which fit well within the overall relationship. 
There was no significant correlation between the wLI-PMd and IT-R, or between the wLI-SMA and IT-R, at either session. There was no significant correlation between the change in wLI and the change in IT-R from Session 1 to Session 2 for any ROI. Finally, significant positive correlations were found between IT-R and $\sum \mathrm{t}$ values for contralesional $\mathrm{M}_{1}$ and SMA at Session 2 but not at Session 1 (data not shown).

\section{Discussion}

Consistent with our hypothesis, the same relationship between the $\mathrm{LI}$ for $\mathrm{M}_{1}$ and maximal tapping ratio prevailed at both fMRI sessions in this cohort. In contrast, no significant correlation was found for PMd and SMA at either time point. Thus the impact of $\mathrm{M}_{1}$ hemispheric balance on finger tapping speed appeared consistent across recovery of motor function over the time period covered in this study.

This study has several limitations. The sample was small and heterogeneous with a mix of dominant and non-dominant hemisphere as well as cortical and subcortical or medullary strokes, so the conclusions should be taken with caution pending replication in a larger sample. Despite the small sample, however, the recovery in motor scores and the parallel increase in $\mathrm{LI}_{-} \mathrm{M}_{1}$ from Session 1 to Session

2 expected from the literature $e^{7,10-12,15}$ were found. Note also that the present sample is comparable in size to previous similar longitudinal investigations ${ }^{7-15}$. Longitudinal fMRI studies of stroke patients are notoriously difficult to carry out due to patient drop-out or unavailability, as well as scanner unavailability and technical failures, among other things. These problems may have created a sample bias here. These same reasons explain why the second fMRI study could not be performed at a fixed time after the first, which is also a limitation.

The correlation between LI-M 1 and IT-R at Session 2 was modest. However, this was a sub-sample of our whole series of 19 chronic-stage patients where the correlation between the IT-R and wLI-M1 was highly significant ${ }^{3}$, which points to the small sample size accounting for it 
Per protocol, the patients were able to perform the required (fixedperformance) fMRI task at both sessions. Other longitudinal designs are possible, such as the patient instructed to do whatever movement they can at Session $1^{12}$, or the task is normalised to maximum ability at both sessions ${ }^{15}$. However, the fixedperformance paradigm is the most widely employed ${ }^{7-11,13,14}$ because it allows direct comparison of the LI for the same motor output over time. The downside is that we could study patients only after they had recovered enough to do the task, which with the above mentioned patient- and scanner-related constraints resulted in Session 1 taking place 10 to 60 days after stroke. Our findings cannot therefore be safely extrapolated to the very early stage of recovery.

We found no statistically significant difference in the correlation between ${ }_{w L I-M}$ and IT-R at both fMRI sessions, which suggests that the same relationship between $M_{1}$ activation balance and motor performance applies throughout the recovery period studied here. In other words, similar functional processes linking the ipsi- and contralesional M1 would be involved across time to produce consistent motor output. Figure 2B illustrates the striking overall relationship between worse hand deficit and more unphysiological LI. Interestingly, as shown in this graph, three subjects (one from Session 1 and two from our previous study) exhibited negative LIs, indicating greater contralesional than ipsilesional $\mathrm{M}_{1}$ activation. This phenomenon has been repeatedly reported before (see reference ${ }^{19}$ for review), and is shown here to correlate with particularly poor motor performance. Because of the fMRI paradigm used, however, we were unable to study more severely affected patients, so cannot say whether this relationship would hold towards the extreme left side of the graph.

The negative findings regarding the IT-R vs wLI-PMd or wLI-SMA relationship at either time-point was expected from our previous report ${ }^{3}$. Previously, only Johansen-Berg et $\mathrm{al}^{4}$ reported a weak trend for a correlation between the LI-PMd and hand motor performance in 10 patients with chronic stroke, but this finding has not been replicated as yet. In their study of intensive arm training in chronic stroke patient, Carey et $\mathrm{al}^{5}$ report a significant increase in LI-PMd between the pre- and posttreatment fMRI studies, but the relationship with individual motor scores is not reported; these authors found the LI-SMA to be on average close to zero at both time points, consistent with our findings. As already mentioned, this lack of clear 
relationship between activation laterality and hand motor performance for PMd and SMA likely reflects the more bilaterally organised function for these two areas in turn due their dense transcallosal connections ${ }^{3}$. The significant positive correlation between $\sum \mathrm{t}$ for contralesional $\mathrm{M}_{1}$ and SMA found at Session 2 are however consistent with our previous report ${ }^{3}$, but they were not present at Session 1.

In conclusion, impaired $\mathrm{M}_{1}$ hemispheric activation balance appears to be associated with poor performance. That this may be partly maladaptive is supported by reports of improvement in hand motor functions following intense training of the affected arm in association with returns of the LI-M towards physiological values $^{5,6}$. The proposed interpretation is that stimulating ipsilateral $M_{1}$ resets the interhemispheric balance which in turn helps recovery. In support of this notion, some recent studies have reported motor improvements following direct stimulation of ipsilesional $M_{1}$ or inhibition of contralesional $M_{1}$ with TMS or tDCS (see reference ${ }^{20}$ for review). Our present findings suggest that such approaches aiming to restore the physiological balance of activation may have the same efficiency over a broad time window after stroke. 


\section{References}

1. Yozbatiran N, Cramer SC. Imaging motor recovery after stroke. NeuroRx. 2006;3:482-488

2. Cramer SC, Crafton KR. Somatotopy and movement representation sites following cortical stroke. Exp Brain Res. 2006;168:25-32

3. Calautti C, Naccarato M, Jones PS, Sharma N, Day DD, Carpenter AT, Bullmore ET, Warburton EA, Baron JC. The relationship between motor deficit and hemisphere activation balance after stroke: A $3 \mathrm{t}$ fmri study. Neuroimage. 2007;34:322-331

4. Johansen-Berg H, Rushworth MF, Bogdanovic MD, Kischka U, Wimalaratna $\mathrm{S}$, Matthews PM. The role of ipsilateral premotor cortex in hand movement after stroke. Proc Natl Acad Sci U S A. 2002;99:14518-14523

5. Carey JR, Kimberley TJ, Lewis SM, Auerbach EJ, Dorsey L, Rundquist P, Ugurbil K. Analysis of fmri and finger tracking training in subjects with chronic stroke. Brain. 2002;125:773-788

6. Takahashi CD, Der-Yeghiaian L, Le V, Motiwala RR, Cramer SC. Robotbased hand motor therapy after stroke. Brain. 2008;131:425-437

7. Calautti C, Leroy F, Guincestre JY, Baron JC. Dynamics of motor network overactivation after striatocapsular stroke: A longitudinal pet study using a fixed-performance paradigm. Stroke. 2001;32:2534-2542

8. Carey LM, Abbott DF, Egan GF, Bernhardt J, Donnan GA. Motor impairment and recovery in the upper limb after stroke: Behavioral and neuroanatomical correlates. Stroke. 2005;36:625-629

9. Feydy A, Carlier R, Roby-Brami A, Bussel B, Cazalis F, Pierot L, Burnod Y, Maier MA. Longitudinal study of motor recovery after stroke: Recruitment and focusing of brain activation. Stroke. 2002;33:1610-1617

10. Jang SH, Cho SH, Kim YH, Kwon YH, Byun WM, Lee SJ, Park SM, Chang $\mathrm{CH}$. Cortical activation changes associated with motor recovery in patients with precentral knob infarct. Neuroreport. 2004;15:395-399

11. Jang SH, Kim YH, Cho SH, Chang Y, Lee ZI, Ha JS. Cortical reorganization associated with motor recovery in hemiparetic stroke patients. Neuroreport. 2003;14:1305-1310

12. Marshall RS, Perera GM, Lazar RM, Krakauer JW, Constantine RC, DeLaPaz RL. Evolution of cortical activation during recovery from corticospinal tract infarction. Stroke. 2000;31:656-661

13. Small SL, Hlustik P, Noll DC, Genovese C, Solodkin A. Cerebellar hemispheric activation ipsilateral to the paretic hand correlates with functional recovery after stroke. Brain. 2002;125:1544-1557

14. Tombari D, Loubinoux I, Pariente J, Gerdelat A, Albucher JF, Tardy J, Cassol E, Chollet F. A longitudinal fmri study: In recovering and then in clinically stable sub-cortical stroke patients. Neuroimage. 2004;23:827-839

15. Ward NS, Brown MM, Thompson AJ, Frackowiak RS. Neural correlates of motor recovery after stroke: A longitudinal fmri study. Brain. 2003;126:24762496 
16. Johansen-Berg H, Dawes H, Guy C, Smith SM, Wade DT, Matthews PM. Correlation between motor improvements and altered fmri activity after rehabilitative therapy. Brain. 2002;125:2731-2742

17. Maldjian JA, Laurienti PJ, Kraft RA, Burdette JH. An automated method for neuroanatomic and cytoarchitectonic atlas-based interrogation of fmri data sets. Neuroimage. 2003;19:1233-1239

18. Tzourio-Mazoyer N, Landeau B, Papathanassiou D, Crivello F, Etard O, Delcroix N, Mazoyer B, Joliot M. Automated anatomical labeling of activations in spm using a macroscopic anatomical parcellation of the mni mri single-subject brain. Neuroimage. 2002;15:273-289

19. Calautti C, Baron JC. Functional neuroimaging studies of motor recovery after stroke in adults: A review. Stroke. 2003;34:1553-1566

20. Bolognini N, Pascual-Leone A, Fregni F. Using non-invasive brain stimulation to augment motor training-induced plasticity. J Neuroeng Rehabil. 2009;6:8 


\section{Figure Legends}

Figure 1: Activation maps (average of the Effect Size maps across the 9 patients) at time-point 1 and time-point 2, with corresponding normal brain MRI cuts (MNI template) for anatomical orientation. One set of axial cuts (left column) and coronal cuts (right column) through the hand motor area (arraows) are shown for illustration. More bilateral M1 activation is present at time point 1 than time point 2, which is quantitated using the Laterality Index in this study (see Methods and Results).

Figure 2: (A): Scatter plot showing the relationship between wLI-M1 and IT-R at the first (red dots, $1 p=0.008$ ) and second (blue dots, $1 p=0.058$ ) fMRI sessions in the same cohort of patients $(n=9)$. The regression lines for both correlations are shown to illustrate that the same relationship was present at both time points (Pearson's $r=-$ 0.796, $1 \mathrm{p}=0.005$; and $\mathrm{r}=-0.550,1 \mathrm{p}=0.06$, respectively; no significant difference). (B): Negative correlation between the wLI-M1 and IT-R for the 19 chronic-stage measurements described in Calautti et $\mathrm{al}^{3}$ (black dots) and the nine Session 1 measurements reported here (red dots); Kendall's $\mathrm{p}=0.002$. 
TABLE 1. Patient demographics, side of hemiparesis, days from stroke at the two fMRI sessions, lesion topography, European Stroke Scale (ESS) score, IT-Max value for the affected hand (AH) and unaffected hand (UH), IT-R, and weighted Laterality Index (wLI) for the primary motor cortex (M1), dorsal premotor cortex (PMd) and supplementary motor area (SMA). See text for details.

\begin{tabular}{|c|c|c|c|c|c|c|c|c|c|c|c|c|}
\hline Pt\# & Sex & $\begin{array}{l}\begin{array}{l}\text { Age } \\
\text { (yrs) }\end{array}\end{array}$ & $\begin{array}{l}\text { Hemi- } \\
\text { paresis } \\
\text { (side) }\end{array}$ & $\begin{array}{l}\text { Days } \\
\text { from } \\
\text { stroke }\end{array}$ & $\begin{array}{l}\text { Lesion } \\
\text { topography }\end{array}$ & ESS & $\begin{array}{l}\text { IT-Max } \\
\text { AH }\end{array}$ & $\begin{array}{l}\text { IT-Max } \\
\text { UH }\end{array}$ & IT-R & wLI-M1 & wLI-PMd & wLI-SMA \\
\hline \multirow[t]{2}{*}{1} & $M$ & 50 & $\mathrm{~L}$ & 48 & L medulla & NA & 34 & 46 & 1.353 & .660 & .774 & .038 \\
\hline & & & & 112 & & NA & 36 & 50 & 1.390 & .757 & .275 & .050 \\
\hline \multirow[t]{2}{*}{2} & $\mathrm{~F}$ & 45 & $\mathrm{~L}$ & 32 & R striato-capsular & 79 & 30 & 50 & 1.666 & .407 & 1.000 & -.041 \\
\hline & & & & 230 & & 89 & 40 & 48 & 1.200 & .767 & .553 & .155 \\
\hline \multirow[t]{2}{*}{6} & $M$ & 71 & $\mathrm{~L}$ & 60 & $\mathrm{R}$ cortical & 84 & 36 & 44 & 1.222 & .555 & -.006 & -.199 \\
\hline & & & & 91 & & 96 & 38 & 46 & 1.211 & .759 & 1.000 & -.554 \\
\hline \multirow[t]{2}{*}{9} & $M$ & 52 & $\mathrm{R}$ & 40 & L corona radiata & 92 & 39 & 45 & 1.154 & .668 & -.487 & .183 \\
\hline & & & & 172 & & 96 & 50 & 50 & 1.000 & .380 & -.827 & -.413 \\
\hline \multirow[t]{2}{*}{10} & $M$ & 52 & $\mathrm{R}$ & 37 & L striato-capsular & 96 & 32 & 38 & 1.188 & .971 & .346 & .620 \\
\hline & & & & 167 & & 100 & 44 & 33 & 0.750 & 1.000 & .847 & .704 \\
\hline \multirow[t]{2}{*}{11} & $M$ & 54 & $\mathrm{~L}$ & 28 & $\mathrm{R}$ corona radiata & 81 & 36 & 44 & 1.222 & .952 & .895 & .021 \\
\hline & & & & 150 & & 81 & 38 & 46 & 1.210 & .983 & 1.000 & -.336 \\
\hline \multirow[t]{2}{*}{15} & $M$ & 65 & $\mathrm{~L}$ & 33 & $\mathrm{R}$ thalamo-capsular & 66 & 38 & 54 & 1.420 & .453 & -.391 & -.643 \\
\hline & & & & 148 & & 80 & 42 & 54 & 1.280 & .828 & .672 & -.686 \\
\hline \multirow[t]{2}{*}{16} & $\mathrm{~F}$ & 65 & $\mathrm{~L}$ & 10 & R striato capsular \& insula & 87 & 30 & 42 & 1.400 & .788 & 1.000 & -1.000 \\
\hline & & & & 90 & & 100 & 42 & 50 & 1.190 & .933 & 1.000 & -.201 \\
\hline \multirow[t]{2}{*}{18} & $\mathrm{~F}$ & 51 & $\mathrm{~L}$ & 35 & R striato-capsular & 74 & 28 & 48 & 1.714 & -.298 & -.647 & -.210 \\
\hline & & & & 166 & & 85 & 32 & 54 & 1.687 & .152 & .988 & .221 \\
\hline
\end{tabular}

ESS: European Stroke Scale (normal score: 100); IT-Max: maximum number of index finger-thumb taps in 15 seconds; AH: Affected hand; UH: unaffected hand; IT-R: Unaffected/Affected IT-Max ratio; N/A: not available. 
Time-point 1
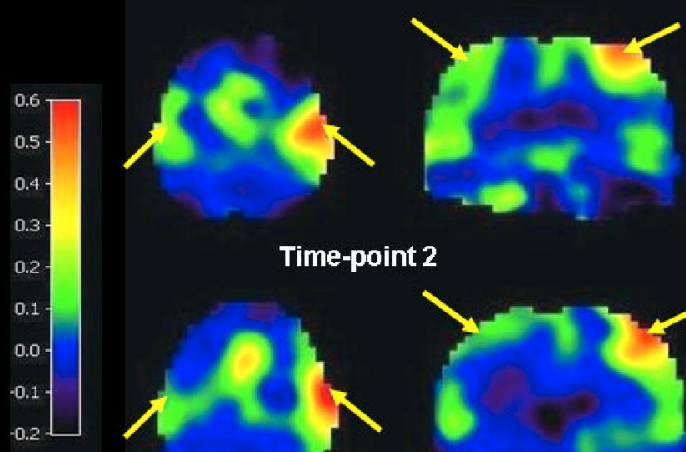

Time-point 2
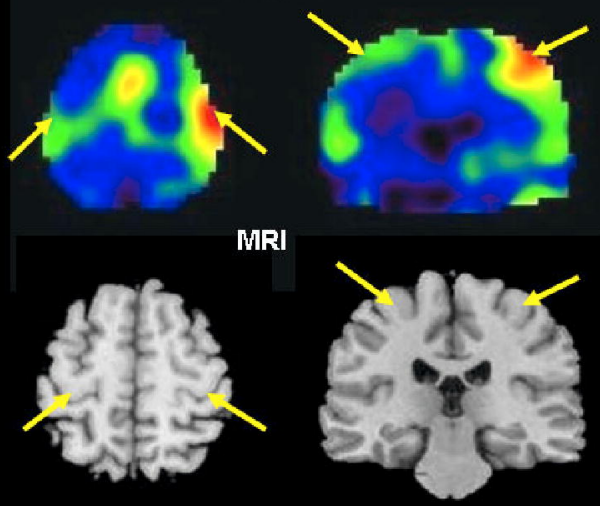

Figure 1 


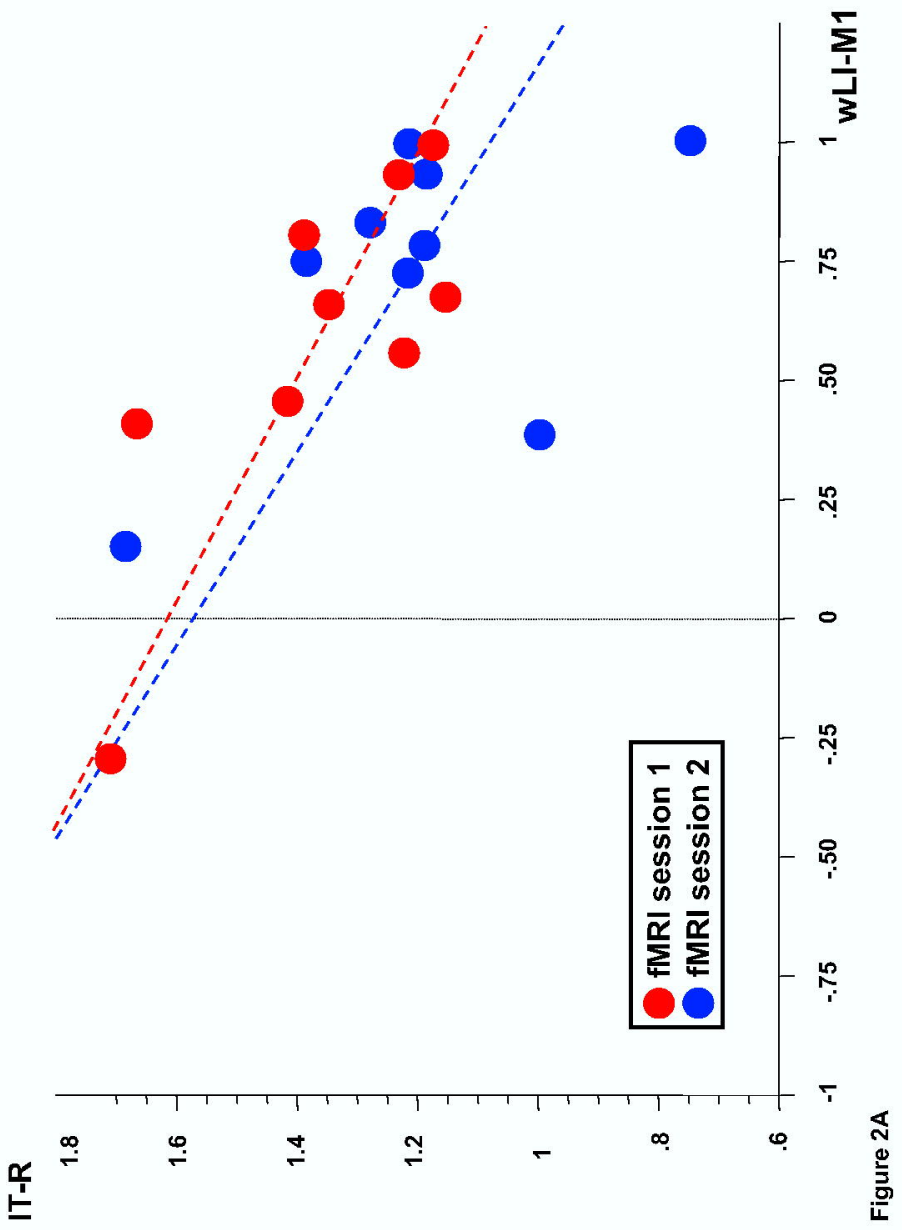




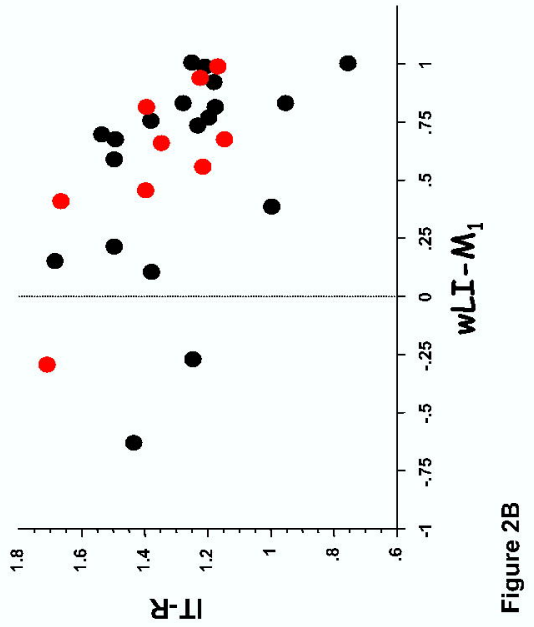

